\title{
Direct Observation of Oxygen Movement in Graphene Oxide-Based Resistive Switching Memory
}

\author{
Sungkyu Kim ${ }^{1}$, Jong Chan $\mathrm{Kim}^{2}$ and Hu Young Jeong ${ }^{3}$ \\ 1. Department of Materials Science and Engineering and NUANCE Center, Northwestern University, \\ Evanston, Illinois, United States \\ 2. School of Materials Science and Engineering, UNIST, Ulsan, South Korea \\ 3. UNIST Central Research Facilities (UCRF) and School of Materials Science and Engineering, UNIST, \\ Ulsan, South Korea
}

The electrically insulating graphene oxide $(\mathrm{GO})$ is one of the emerging materials due to its unique structure and controllable properties. The electrical properties of synthesized GO flake bonded various oxygen containing groups could be tuned suitably by reduction process. Because of this, GO has great attention as an insulating and conducting films in nano-electronic devices such as the non-volatile memory (NVM) [1]. Previously, we reported the resistive switching behavior with excellent properties in GO-based NVM [2, 3]. However, the effect of the local oxygen diffusion in GO films on conduction path is not clearly demonstrated. Here, we report on the reduction of GO films caused by oxygen movement under an electric bias using in-situ transmission electron microscope (TEM) technique.

The cross-bar type Pt/GO/Pt memory device composed of spin-coated GO films sandwiched between Pt electrodes was fabricated. The current flows in the direction of the arrow, and the resistance decreased after the negative sweep, as shown in a current-voltage (I-V) curve (Figure 1a). Although I-V curve is similar to the conventional resistive switching devices, Pt/GO/Pt device was destroyed during the negative sweep (marked with a red circle) due to the formation of bubble. Similar phenomena, gas eruption causes by oxygen ions drift, have been reported in $\mathrm{Pt} / \mathrm{TiO}_{2} / \mathrm{Pt}$ device [4]. Figure $1 \mathrm{~b}$ shows cross-sectional TEM image of the edge of bubble in the inset of Figure 1a (marked with a blue square) after the SET process. GO films below the top electrode (region 2) has a darker contrast than the side region (without the top electrode). It means that GO films were highly reduced by electric bias. Crosssectional high resolution TEM images and its fast Fourier transform (FFT) patterns show the reduction of GO films by diffusion of oxygen ions (Figure 1c and 1d). Irregularly oriented GO sheets with oxygen groups were transformed into densely stacked structures. The oxygen ions moved from region 2 to region 1 and supersaturation of oxygen ions caused the bubble destruction. To understand the dynamic motion of oxygen ions in GO films, we utilized in situ TEM under an electric bias condition. Figure 2a shows I-V curve of Pt/GO/Pt device prepared by focused ion beam (FIB). The current increase rapidly during the negative sweep, and it shows current ratio of 21.4 times. Figure $2 b-2 d$ sequentially show the transition of $\mathrm{Pt} / \mathrm{GO} / \mathrm{Pt}$ device under the electric bias. The device did not show the bubble destruction due to the relatively low amounts of oxygen ions and ultra-high vacuum condition compared to ex-situ experiments. Cross-sectional HRTEM images in Figure $2 \mathrm{e}$ and $2 \mathrm{f}$ are consistent with ex-situ results. When the negative bias was applied to the top electrode, the pristine GO films with an amorphous-like structure changed to reduced GO films because the negatively charged oxygen ions moved to other regions or escaped out of GO films. From cross-sectional TEM images and in situ experiment, we demonstrate that oxygen movement in GO films makes the electrical path in $\mathrm{Pt} / \mathrm{GO} / \mathrm{Pt}$ memory device [5]. 


\section{References:}

[1] Samuele Prro et al, Carbon 85 (2015), p. 383.

[2] Sung Kyu Kim et al, Advanced Functional Materials 25, (2015), p. 6710.

[3] Sung Kyu Kim et al, Advanced Functional Materials 26, (2016), p. 7406.

[4] J Joshua Yang et al, Nanotechnology 20, (2009), p. 215201.

[5] This work is supported by NRF-2014R1A1A2058713.
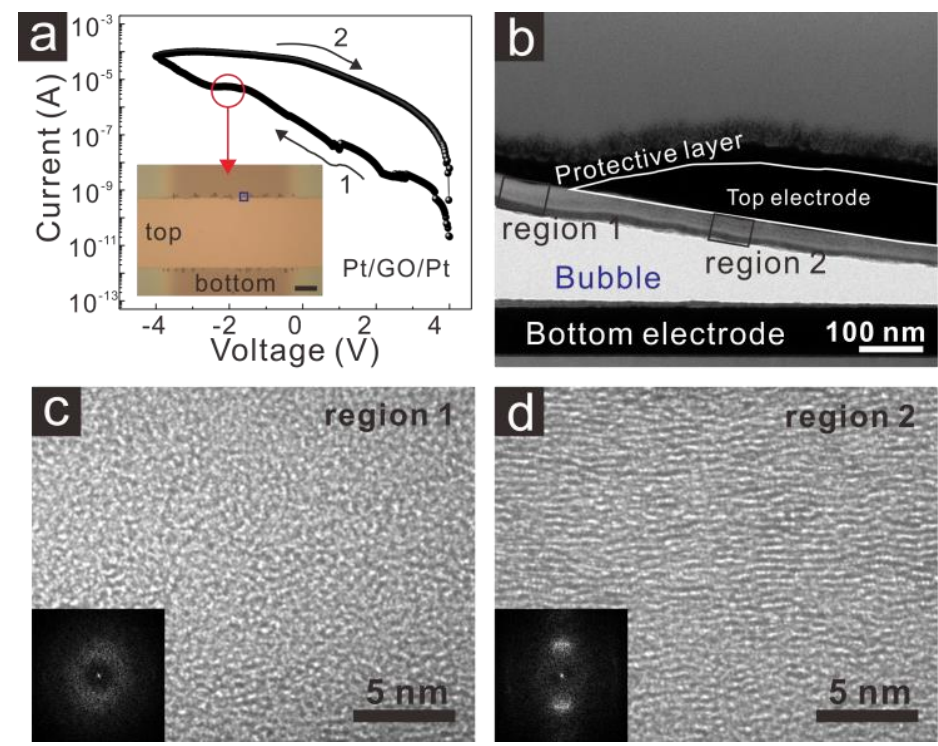

Figure 1. (a) Typical current-voltage (I-V) curve of $\mathrm{Pt} / \mathrm{GO} / \mathrm{Pt}$ device. The inset is the optical microscope image of bubbles. (b) Cross-sectional TEM images of the bubble after the SET process. (c) and (d) are HRTEM images of each rectangular region in (b). The each inset shows FFT pattern of GO layer.
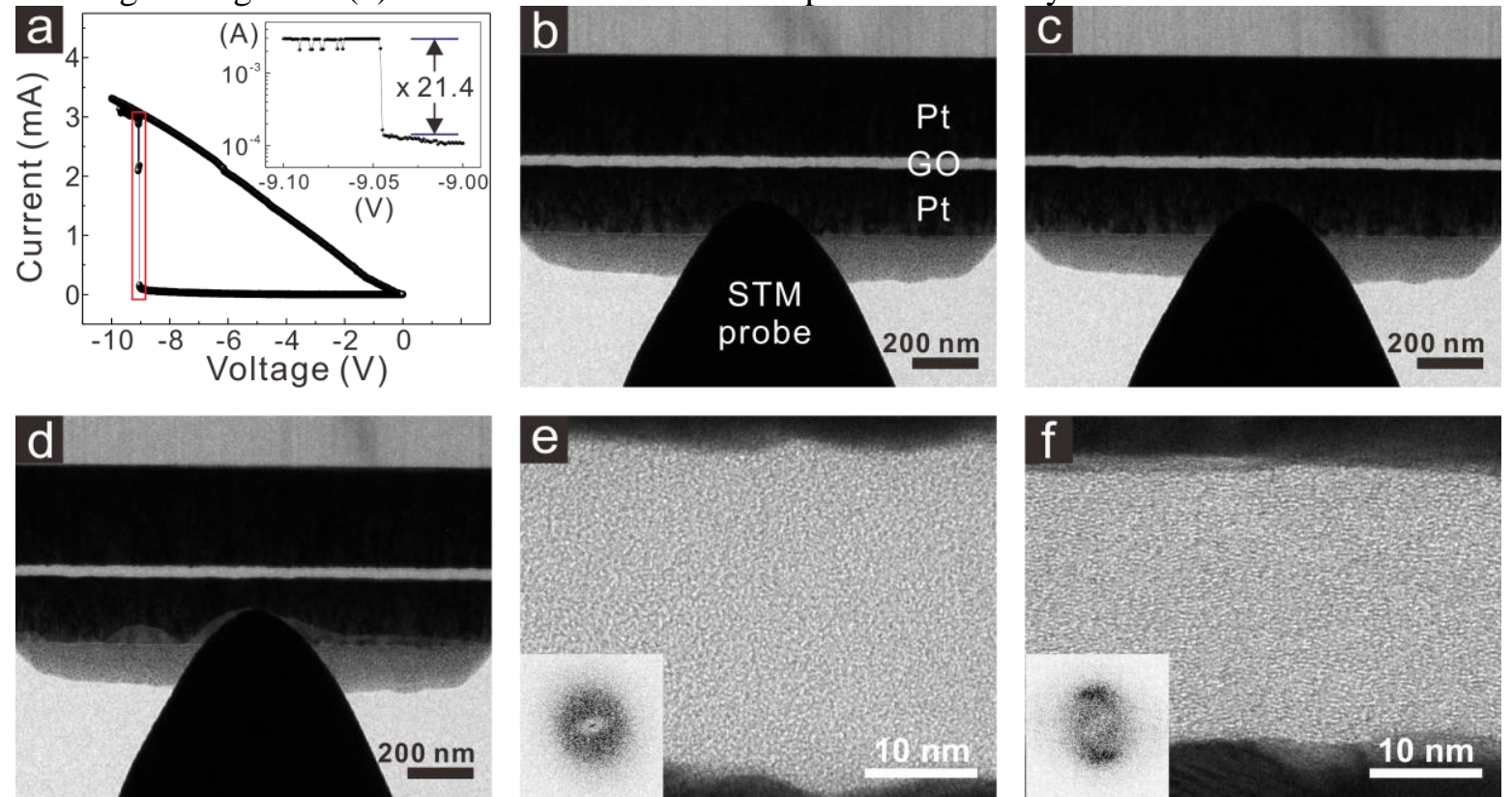

Figure 2. (a) I-V curve of $\mathrm{Pt} / \mathrm{GO} / \mathrm{Pt}$ device under in-situ TEM characterization. The inset shows a magnified electrical property at the SET process in the red rectangular region. (b-d) Sequentially captured real-time crosssectional BFTEM images under the electric bias. Cross-sectional HRTEM images of (e) the OFF state and (f) the ON state. The each inset shows FFT pattern of GO layer. 